\title{
Multi-evidence fusion recognition of ship targets in sea battlefield's remote sensing images
}

\author{
Yu An ${ }^{1,2}$, Xiaofei Wang, Xuezhi Xia and Lin Li
}

\begin{abstract}
Reliably recognizing ship targets in the sea battlefield has become an increasingly pressing need, as the capabilities for image acquisition are growing rapidly. In the research, a modified ship targets fusion recognition model based on the Dempster-Shafer evidence theory is proposed. The algorithm firstly detects ship targets from the sea battlefield's remote sensing image. Then extracts the multiple image features of these target candidate areas as the evidence to recognize the ship targets. Finally, recognizes the ship targets from the image using the Dempster-Shafer evidence theory based on multiple ship features, and sends the recognition result. Experiment show that this method can be used to reliably and effectively recognize targets information in the sea battlefield.
\end{abstract}

Keywords: target recognition; Dempster-Shafer evidence; ship features; remote sensing image

\section{Instruction}

As one of the main areas of operations of modern war, Sea battle field situation vary from minute to minute in battle. Ship targets are the key targets of the maritime monitoring and wartime attack. Rapid and accurate identification of ship sea battlefield tactics and provide support for decision-making for commanders, which is greatly related to the success or failure of the battle. Many judging methods of warship target intention in a sea battle, they are built on the basis of the target recognition. In modern sea battles, in order to make the situation assessment and battlefield decision, we should first solve how to recognize accurately different kinds of battle ships in the distance.

\footnotetext{
${ }^{1} \mathrm{Yu}$ An $(\bowtie)$

College of Computer Science and Technology, Harbin Engineering University. Harbin, China e-mail: anyu723@126.com

${ }^{2} \mathrm{Yu}$ An

Wuhan Digital Engineering Institute. Wuhan, China
} 
Previous research for ship target recognition is mainly based on the synthetic aperture radar or forward-looking infrared radar image data, these methods are using the difference of Ship Superstructure to recognize the type of ship targets[1,2,3]. In recent years, with the development of satellite remote sensing technology, emerging a number of imaging satellite with high spatial resolution and short revisit period, which provides abundant data source maritime reconnaissance and target monitoring. Using optical remote sensing image to recognize ship targets becomes possible. For example, Zhang[4] use the support vector machine technology to recognize the ship targets in Quick Bird satellite data, Lan et al[5] propose a modified Zernike moments model for recognizing the large warship in aerial images.

Unlike the methods proposed in the above, focusing on the problem of low recognition rate which is because of the ship image target recognition with imprecise, uncertain, incomplete etc., a new research has been done based on the multifeature fusion recognition idea. With resolution better than $4 \mathrm{~m}$ optical remote sensing image data as an object of study, this paper proposes a multi-evidence fusion recognition method based on the ship features. The method consist of the following steps: Firstly, detecting and binarizing the targets area in the image, for a single feature information is insufficient or easily failure to recognize the ship targets, in order to get enough useful complementary information, extracting the length, width, area and many features from the targets slice as the evidence sources; Then, establishing the Dempster-Shafer(D-S) fusion recognition framework and computing the value of Basic Belief Assignment of each feature; Finally, using D-S combination rule to complete the decision level fusion and output ship target recognition results.

\section{Ship feature selection and extraction}

\subsection{Ship feature selection}

Spatial distribution characteristics of the ship targets mainly describe their physical properties, including size, brightness, and shape feature parameters. Size and shape features emphasize distinguish objects based on geometric parameters, which can reflect differences in the nature of ship in the military field, such as frigate and aircraft carrier. In order to be able to better and more accurate recognition the ship targets, firstly need to analyze the different types of ship targets, then select the features which can be easy to distinguish the ship targets. In the high resolution remote sensing images, the size and shape features of targets can be easy to 
extraction. In this paper, select the following features as the evidence for recognition of ship targets.

Size features: ship length $f_{1}$, ship width $f_{2}$, ship aspect ratio $f_{3}$, ship surface area $f_{4}$.

Shape features: ship bow $f_{5}$, ship stern $f_{6}$, ship junction $f_{7}$.

\subsection{Ship feature extraction and definition}

In order to extract the ship features from images, we need to binarize target image $f(x, y)$ and calculate the moment features of image. The $(p+q)$ order origin moment and the $(p+q)$ order central moments are defined as:

$$
\begin{gathered}
m_{p q}=\sum_{i} \sum_{j} i^{p} j^{q} f(i, j) \\
\mu_{p q}=\sum_{i} \sum_{j}(i-\bar{x})^{p}(j-\bar{y})^{q} f(i, j)
\end{gathered}
$$

Where $(\bar{x}, \bar{y})$ is the centroid coordinate of target image $f(x, y)$.

Normalized central moments:

$$
\begin{gathered}
U_{x x}=\frac{\sum_{i=1}^{N}\left(x_{i}-\bar{x}\right)^{2}}{U_{00}} \\
U_{y y}=\frac{\sum_{i=1}^{N}\left(y_{i}-\bar{y}\right)^{2}}{U_{00}} \\
U_{x y}=\frac{\sum_{i=1}^{N}\left(x_{i}-\bar{x}\right)\left(y_{i}-\bar{y}\right)}{U_{00}}
\end{gathered}
$$

Target spindle direction is defined as:

$$
\theta= \begin{cases}\arctan \left(2 * U_{x y}, U_{x x}-U_{y y}+\sqrt{\left(U_{x x}-U_{y y}\right)^{2}+4 * U_{x y}^{2}}\right) & U_{x x} \geq U_{y y}(6) \\ \arctan \left(U_{y y}-U_{x x}+\sqrt{\left(U_{y y}-U_{x x}\right)^{2}+4 * U_{x y}^{2}}, 2 * U_{x y}\right) & U_{x x} \leq U_{y y}\end{cases}
$$

Where $\theta$ is the angle between target spindle and axis, $\theta \in\left(-\frac{\pi}{2}, \frac{\pi}{2}\right)$. When including geographic information in image, $\theta$ can be express ship heading.

Rotate target spindle to the horizontal and get the target minimum bounding rectangle. 
$f_{1}$ is defined as the length of bounding rectangle.

$f_{2}$ is defined as the width of bounding rectangle.

$f_{3}$ is defined as the aspect ratio of bounding rectangle.

Four kinds of ship target aspect ratio statistics are as follow (Table 1): Table 1 Aspect ratio tables of warships

\begin{tabular}{cc}
\hline Warship & Aspect Ratio \\
\hline Destroy(Cruiser) & $8.5: 1 \sim 10.5: 1$ \\
Frigate & $8.1: 1 \sim 9.5: 1$ \\
Aircraft carrier & $5.5: 1 \sim 8.2: 1$ \\
\hline
\end{tabular}

Obviously, the different uses of the ship has different aspect ratio interval. Therefore, it can recognize different types of ship by using this feature, especially recognizing the aircraft carrier from the fleet.

$f_{4}$ is defined as the sum of detection target region pixels. Being much larger than other ships, aircraft carriers can be effectively distinguished from other ships based on $f_{4}$. The actual area of the ship target is the sample interval of pixel multiplied by the total number of target pixels.

According to the characteristics of modern sea battle, military action mainly focus on recognizing destroyers, cruisers, frigates and aircraft carriers in the sea battlefield. Ship bow, body and stern have obvious geometry features. For target recognition, they can be simplified as triangle, rectangle and trapezoid (figure 1).

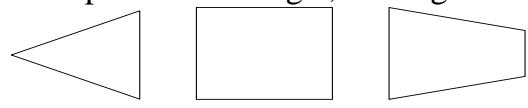

Fig. 1 Simplified features of ship target

In order to describe effectively the ship target's bow and stern shape characteristics, the target is divided into areas. Each area size is as close as possible to the size of bows or sterns. In the actual ship shape feature extraction, it is generally thought that image resolution should be better than $4 \mathrm{~m}$. Considering that a bow or a stern in general is about $1 / 8 \sim 1 / 3$ of the whole ship, the area number divided is $3 \sim 8$.

To describe accurately the shape characteristics of bows and sterns and ensure the algorithm efficiency, the area's number is 5 and marked $A_{1}-A_{5}$ as follows.

\begin{tabular}{|l|l|l|l|l|}
\hline $\mathrm{A}_{1}$ & $\mathrm{~A}_{2}$ & $\mathrm{~A}_{3}$ & $\mathrm{~A}_{4}$ & $\mathrm{~A}_{5}$ \\
\hline
\end{tabular}

Fig. 2 Example of a ship slice

As figure 2 shows, bows and sterns are represented by $A_{1}$ and $A_{5}$. In this paper, the shapes of bows and sterns are abstracted to combination of triangle and 
trapezoid. In ship targets recognition, the junction feature of ship targets is the number of junctions of bows and sterns.

$f_{5}, f_{6}$ and $f_{7}$ are defined as follows(Table 2).

Table 2 Ship shape features and Junction features

\begin{tabular}{cccc}
\hline Warship & \multicolumn{3}{c}{ Features } \\
\cline { 2 - 4 } & bow $\left(f_{5}\right)$ & stern $\left(f_{6}\right)$ & junction $\left(f_{7}\right)$ \\
\hline Destroy(Cruiser) & triangle & trapezoid & 7 \\
Frigate & triangle & trapezoid & 7 \\
Aircraft carrier & trapezoid & trapezoid & 8 \\
\hline
\end{tabular}

\section{Multi-feature fusion recognition of ship targets based in D-S evidence theory}

Ship target area, length, width, aspect ratio, shape and junction feature are independent of each other. It is difficult to meet target recognition requirement depending on a single feature. For example, a target whose shape is meet requirement is not the type intended to identify but there is a possibility of belonging to the type. In order to use effectively various features to improve target recognition rate, D-S evidence theory is applied to fuse the information from different features[6]. The decision module can find the target's type attribute and confidence level. Figure 3 shows the framework of multi-feature fusion and recognition arithmetic.

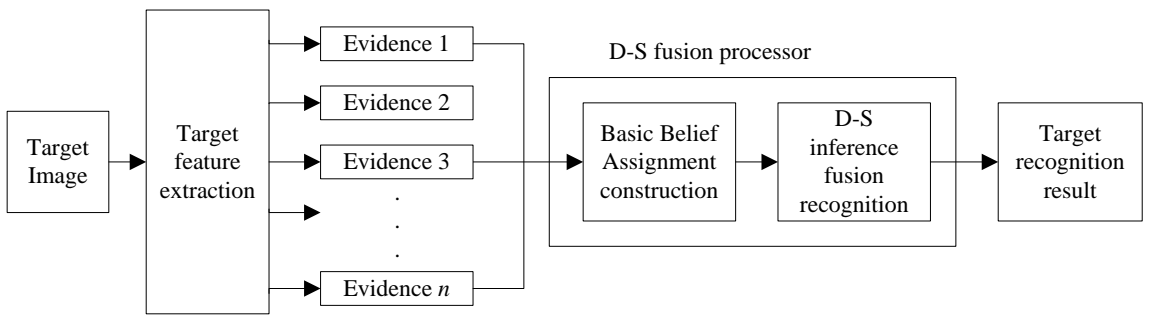

Fig. 3 The framework of multi-feature fusion and recognition arithmetic

Let $\Theta$ be a universe which includes all possible values of target type in the sea battlefield. All elements in $\Theta$ are mutually exclusive. So $\Theta$ is a recognition framework of the sea battlefield's target type. Define a map $m: 2^{\Theta} \rightarrow[0,1]$, it satisfies:

$$
\left\{\begin{array}{c}
m(\phi)=0 \\
\sum_{A \in 2^{\Theta}} m(A)=1
\end{array}\right.
$$


Where $m$ is the basic belief assignment of $\Theta, m(A)$ is the value of basic belief assignment of $A$. When $A \neq \Theta, m(A)$ represents the precise level of the proposition $A$ 's trust; When $A=\Theta, m(\Theta)$ is the degree of uncertainty.

This fusion algorithm is focus on the targets in the sea battlefield, so the target fusion recognition frame is defined as a set of warships. Surface targets are mainly destroyers, cruisers, frigates and aircraft carriers in the sea battlefield. In the entire aircraft carrier battle group, destroyers and cruisers have the similar size, position of the fleet and performs the similar functions. Therefore, destroyers and cruisers are classified as a class in the multi-feature fusion recognition framework. The framework of target recognition contains three elements (destroyer or cruiser, frigate, aircraft carrier), so $\Theta$ can be expressed as a set of $\Theta=\{D, F, C\}$. In the set, $D$ represents destroyers and cruisers, $F$ represents frigates and $C$ represents the aircraft carriers. Using the basic belief assignment function to describe the uncertainties in the process of target recognition, we define a basic belief assignment function $m_{k}$ which meet the following conditions:

$$
\begin{gathered}
m_{k}(\phi)=0 \\
m_{k}(D)+m_{k}(F)+m_{k}(C)+m_{k}(\Theta)=1
\end{gathered}
$$

Where $\mathrm{k}$ is the number of target recognition features; $m_{k}(D), m_{k}(F)$ and $m_{k}(C)$ are the value of basic belief assignment of the different kinds of targets in the image, which are computed by image features; $m_{k}(\Theta)$ represents the degree of uncertainty in the decision process.

In order to get the basic belief assignment value of multi-feature fused, we use dempster fusion rule to process each feature confidence value. Dempster fusion rule:

Let $m_{1}, m_{2}, \ldots, m_{k}$ represents the value of basic belief assignment in the identification framework $\Theta$, which comes from different sources of evidence; through orthogonal and composite the fused basic level of confidence can be gotten.

$$
m(A)=\left(m_{1} \oplus m_{2} \oplus \ldots \oplus m_{j}\right)(A)=\frac{\sum_{\cap A_{i}=A}\left[\prod_{1 \leq j \leq k} m_{j}\left(A_{i}\right)\right]}{1-\sum_{\cap A_{i}=\phi}\left[\prod_{1 \leq j \leq k} m_{j}\left(A_{i}\right)\right]}
$$

Where $A, A_{i} \subseteq \Theta ; m$ is the basic belief assignment function which is constructed by multi-feature evidence.

According to the calculation result on above, the following two formulas are the Bel(belief function) and the Pls(plausible function).

$$
\begin{aligned}
& \operatorname{Bel}(A)=\sum_{A_{i} \subset A} m\left(A_{i}\right) \\
& \operatorname{Pls}(A)=\sum_{A_{i} \cap A \neq \phi} m\left(A_{i}\right)
\end{aligned}
$$


Where $A, A_{i} \subseteq \Theta ; \operatorname{Bel}(A)$ denotes $A$ which is really trust; $P l s(A)$ denotes $A$ that is really not negate degrees. For $\forall A \subset \Theta, A$ 's confidence level equal to the sum of the value of basic belief assignment which is computed by all subsets in $A$. From the above, target type verdict probability interval is $[\operatorname{Bel}(A), \operatorname{Pls}(A)]$.

In the fusion recognition framework, different target's Bel and Pls can be expressed as:

$$
D:\left\{\begin{array}{c}
\operatorname{Bel}(D)=m(D) \\
P l s(D)=m(D)+m(\Theta)
\end{array}\right.
$$

Where $D$ probability interval is $[\operatorname{Bel}(D), P l s(D)], D \in \Theta$.

$$
F:\left\{\begin{array}{c}
\operatorname{Bel}(F)=m(F) \\
\operatorname{Pls}(F)=m(F)+m(\Theta)
\end{array}\right.
$$

Where $F$ probability interval is $[\operatorname{Bel}(F), P l s(F)], F \in \Theta$.

$$
C:\left\{\begin{array}{c}
\operatorname{Bel}(C)=m(C) \\
P l s(C)=m(C)+m(\Theta)
\end{array}\right.
$$

Where $C$ probability interval is $[\operatorname{Bel}(C), P l s(C)], C \in \Theta$.

\section{Instance}

Experimental research environment: AMD Athlon Dual Core Processor $4600+$ 2.21GHz, 4.0GB RAM, Window XP, Matlab 2009a. The experimental data comes from Google Earth, the image resolution is better than $4 \mathrm{~m}$.

First step is to detect targets in the image and compute the target features as part 2 description. The following is to use the results of part 2 as evidence and put all evidence into the multi-feature fusion framework as part 3 description. With the multi-feature fusion decision, Bel and Pls are applied to calculate the confidence interval of targets. Final step is to get the target type information and its confidence.

\begin{tabular}{|c|c|c|c|c|}
\hline \multirow{2}{*}{$\begin{array}{l}\text { Target } \\
\text { Num }\end{array}$} & \multirow[t]{2}{*}{ Target Image } & \multicolumn{3}{|c|}{ Warship } \\
\hline & & Destroy(Cruiser) & Frigate & $\begin{array}{c}\text { Aircraft carri- } \\
\text { er }\end{array}$ \\
\hline 1 & & $3.9 \%$ & $2.5 \%$ & $93.6 \%$ \\
\hline 2 & & $32.3 \%$ & $66.9 \%$ & $0.8 \%$ \\
\hline 3 & & $69.6 \%$ & $26.7 \%$ & $3.7 \%$ \\
\hline
\end{tabular}

Table 3 is the target recognition results by using our algorithm. Table 3 Target recognition results 


\begin{tabular}{l|lll}
\hline 4 & $8.6 \%$ & $1.2 \%$ & $91.2 \%$ \\
& & & \\
& & & \\
& $35.6 \%$ & $63.6 \%$ & $0.8 \%$ \\
& & & \\
\hline
\end{tabular}

From the table 3 , the confidence of target 1 and target 4 is over $85 \%$, which concern target is aircraft carriers. It is determined to be targets of aircraft carrier. The confidence of target 2 and target 5 is over $60 \%$, which concern target is frigates, so targets of frigate can be found. The confidence of target 3 is over $65 \%$, which concern target is destroys or cruisers, it can be determined targets of destroy or cruiser. Comparing the experimental results with the image data, our algorithm can correctly identify ship targets.

\section{Conclusions}

This paper proposes a ship target recognition algorithm which is suitable for sea battlefield. The research extracts the feature information from target image and use multi-feature of the ship to establish the multi-feature evidence fusion frame based on D-S evidence theory. Because single feature is difficult to recognize the target and make a wrong decision possibly, By the multi-feature evidence fusion we can improve the ability to recognize the target. The experimental results show that our algorithm can reliably and accurately identify ship targets in the sea battlefield and improve the recognition ability of the system.

\section{References}

1. Zabidi M M A, Mustapa .I, et al. Embedded vision systems for shin recognition [C]. IEEE TCENCON. Washington DC, USA: IEEE Computer Society, 2009:1-5.

2. Luo Q, Khoshgoftaar T M, et al. Classification of ships in surveillance video [C]. IEEE International Conference Information Reuse and Integration. Washington DC, USA: IEEE Computer Society, 2006: 432-437.

3. Luo Q, Khoshgoftaar T M, et al. Classification of ships in surveillance video [C]. IEEE International Conference Information Reuse and Integration. Washington DC, USA: IEEE Computer Society, 2006: 432-437.

4. Zhang Z. A Study on Harbor target Recognition in High Resolution Optical Remote Sensing Image [D]. Hefei: University of Science and Technology of China, 2009.

5. Lan J, Wan L. Automatic ship target classification based on aerial images [C]. Proceedings of SPIE. Bellingham Wash: SPIE, 2009, 7156(12): 1-10

6. A. P. Dempster. Upper and Lower Probabilities Induced by a Multi-valued Mapping[J]. Ann. Math. Statist. Volume 38, Number 2 (1967), 325-339. 